\title{
液态金属用作润滑剂的研究现状与展望"
}

\author{
徐明宇陈渭 \\ (西安交通大学现代设计及转子轴承系统教育部重点实验室 西安 710049)
}

\begin{abstract}
摘要: 液态金属作为一种新型材料, 通常是指熔点低于 $200{ }^{\circ} \mathrm{C}$ 的低熔点合金, 是一种不定型的、可流动的金属, 其中室温液 态金属的熔点更低, 在室温下即呈液态, 正是因为这种不定型的液体形态, 使其有着很好的流动性、导热性、热稳定性等性 能, 目前已经应用于诸多领域。国外在 20 个世纪就已经开始了对液态金属润滑性能的研究，但国内对液态金属用作润滑剂 的研究目前还在起步阶段。介绍了液态金属的制备过程，从液态金属的润滑性能、液态金属的润滑机理和液态金属轴承的工 作性能三大方面出发, 总结了液态金属用作润滑剂的研究现状, 并且提出了我国今后在该研究上的努力方向以及未来使用液 态金属作润滑剂要解决的几个问题和技术。
\end{abstract}

关键词: 液态金属; 润滑; 工作性能; 摩擦; 磨损; 发展

中图分类号: TH117

\section{Research Progress and Prospect of Liquid Metals Used as Lubricants}

\author{
XU Mingyu CHEN Wei \\ (Key Laboratory of Education Ministry for Modern Design and Rotor-bearing System, \\ Xi'an Jiaotong University, Xi'an 710049)
}

\begin{abstract}
As a new material, liquid metal usually refers to a low melting point alloy with a melting point below $200{ }^{\circ} \mathrm{C}$. It is an amorphous, flowable metal. The liquid metal at room temperature has a lower melting point and is liquid at room temperature. Because of this amorphous liquid form, it has good fluidity, thermal conductivity, thermal stability and other properties. It has been used in many fields. It has been studied on the lubrication properties of liquid metal in foreign countries since the 20th century, but the research on the use of liquid metals as a lubricant in China is still in its infancy. The preparation process of liquid metals is introduced. From the three aspects of lubrication performances of liquid metals, lubrication mechanisms of liquid metals and working performances of liquid metals bearings, the research status of liquid metal used as lubricant is summarized, and the direction of the research and the problems and technologies to be solved in the future using liquid metal as a lubricant in China is proposed.
\end{abstract}

Key words: liquid metals; lubrication; working performance; friction; wear; prospect

\section{0 前言}

液态金属是一种不定型的、可流动的金属，正 是因为这种不定型的液体形态，使其具有极佳的电 性能和热力学性能, 目前广泛应用于电池、电力设 备、生物医疗、3D 打印和计算领域 ${ }^{[1-3]}$, 我国清华 大学的刘静团队 ${ }^{[4-5]}$ 通过设计实验证明了液态金属 可以作为未来软体机器人新材料的可能, 此项研究 使得液态金属的应用领域进一步得到拓宽。

* 国家重点研发计划资助项目(2017YFC0111500)。20190702 收到初稿, 20200205 收到修改稿
随着现代工业的不断进步和发展，机构的运行 环境要求越来越苛刻 ${ }^{[6]}$, 因此对机械系统中所使用 的润滑剂提出了更高的要求。荷兰飞利浦医疗机构 生产的 IMRC 球管 ${ }^{[7]}$, 将产生医疗诊断用 X 射线的 $\mathrm{CT}$ 球管从固定阳极改为旋转阳极, 并使用以液态金 属作为润滑剂的螺旋槽阳极轴承, 不但改善了 CT 球管的使用性能, 还拓宽了研究视野, 这也是目前 液态金属用于流体动压润滑的成熟案例。早在 1963 年 BUCKLEY 和 JOHNSON ${ }^{[8]}$ 就研究过富含镓的薄 膜在空气和真空环境中作为润滑剂的性能, 发现真 空中的摩擦和磨损比空气中低。在接下来的实验中, KOSTORNOV 等 ${ }^{[9]}$ 又发现液态金属可以有效润滑由 镍和铬基合金纤维材料制成的轴承。同样在 
$\mathrm{NASA}^{[10]}$ 早期工作中发现, 当用于润滑不锈钢和镍 时, 在镓、铟、锡共晶附近的合金具有良好的摩擦 学性能。德克萨斯大学的 PERSAD 等 ${ }^{[11]}$ 研究了在电 枢一轨道界面处存在常见的液态金属现象, 液态铝 通过相对冷的铜轨淬火, 形成淬火金属沉积物, 在 具有足够厚的液态铝熔体润滑的固体电枢轨道中, 轨道不会腐蚀, 该研究表明, 找到合适的润滑剂与 配对材料, 不但可以降低摩擦, 还可以起到一定防 止腐蚀的作用。这些发现都在一定程度上启发了现 在人们对液态金属用作润滑剂的研究。

但是在早期液态金属用作润滑剂的研究中, 人 们只是停留在发现液态金属所体现出有效的润滑性 能上, 对于液态金属的润滑机理并没有做深入的调 查。对于液态金属用作润滑剂的研究, 国内也是处 在起步阶段。本文主要从近几年来国内外对液态金 属的润滑性能研究和润滑机理研究, 以及对实际应 用中液态金属轴承工作性能的分析出发, 论述了液 态金属作为润滑剂的研究现状, 并对液态金属作为 润滑剂的未来发展做了展望。

\section{1 液态金属的制备}

目前关于液态金属的使用与研究主要集中在室 温液态金属。对于非室温液态金属, 目前主要的实 际应用为高速增殖反应堆的钠㬌中所使用的纯钠金

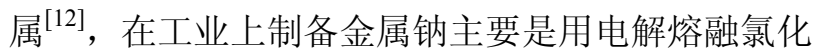
钠的方法。室温液态金属在室温下即为液态, 而在 实际应用中多使用室温液态金属的合金，其制备过 程完全不同于非室温液态金属, 大体制备过程如下: 以高纯度的金属作为原材料, 按照重量的百分比选 取适量材料到容器中混合, 用来混合的容器是要预 先用去离子水清洗过的, 原材料混合后, 加热到一 定温度直至金属熔化, 再进行轻微摚拌, 冷却至室 温后即可得到液态金属。

此方法广泛应用于镓-铟-锡室温液态合金的制 备, 但是要格外注意的是镓基液态金属在空气中非 常容易氧化,而氧化镓会对镓基液态金属的黏度和表 面张力产生影响 ${ }^{[13-14]}$, 所以混合加热过程最好在真空 中进行。若没有真空条件, 可以进行除杂步骤, 即用 一定浓度的 $\mathrm{NaOH}$ 溶液与制备好的液态金属合金按 比例混合后充分摚拌, $\mathrm{NaOH}$ 溶液与液态金属互不相 容, 且液态金属密度较大, 静置后会分层, 用胶头滴 管取下层溶液即可得到除杂后的液态金属。

清华大学教授刘静与他的团队在国内属于较早 也较成熟地研究液态金属的科研团队, 他们用此方 法制备出了质量比为 $75.5: 24.5$ 的镓铟合金 ${ }^{[15]}$, 同
时经过试验研究表明, 在液态金属的制备过程中, 可以通过控制摚拌时间和旋转速度来改变合金的含 氧量, 以获得不同黏度的液态金属。

总之, 制备液态金属主要采用的是熔炼制备工 艺, 即将所需的不同金属材料按照一定的百分比, 通过控制温度加以摚拌使其充分融合，从而形成新 的合金材料。根据金属材料成分和配比的不同，最 终形成的液态金属材料将具备不同的功能与特性, 比如与共晶 Ga-In-Sn 液态金属相比, 共晶 Ga-Sn-Zn 液态金属的熔化温度和表面张力更高, 而热膨胀系 数、密度、黍度、导电率和导热系数更低 ${ }^{[16]}$ 。

\section{2 液态金属润滑性能的研究现状}

\section{1 液态金属的减摩性能}

目前研究液态金属减摩性能的主要实验过程 为: 通过改变摩擦副的配副材料和试验载荷, 采用 球一盘或者栓一盘接触的摩擦方式，在不同温度、 频率、振幅和时间下进行实验对比，或保持上述参 数不变与其他润滑条件下的摩擦结果进行实验对 比, 之后使用表面轮廓仪测得磨损体积和三维形貌, 以计算出磨损率。此方法相对简单, 也是国内研究 液态金属减摩性能最常用的方法。

国内研究液态金属减摩性能的主要以西北工业大 学刘维民教授的团队 ${ }^{[17-20]}$ 为代表, 他们使用 SRV-IV型 摩擦磨损试验机(德国 Optimol 公司)和 MicroXAM-800 非接触表面轮廓仪，对镓基液态金属的减摩性能做 了初步评价, 发现相较于 Ga-In 二元组分, Ga-In-Sn 三元组分的液态金属具有更佳的减摩效果, 并且通 过改变摩擦副的材料, 发现室温镓基液态金属作为 润滑剂时的减摩性能与摩擦副的材料密切相关, 在 电子显微镜下能够清晰看出, 没有液态金属润滑的 材料表面在较低载荷下呈现明显的凹槽和分层。但 是目前研究液态金属减摩性能的实验相对单一, 没 有深入研究不同质量分数的液态金属, 也没有用更 多变的摩擦副材料进行试验。在接下来的研究中, 团队为了观察摩擦材料深层次的结构变化, 在观察 完表面形貌后, 沿着滑动方向进行了切割, 从而进 一步观察, 试验表明镓基液态金属可以作为载流润 滑剂, 并且与无电流对比, 施加电流可以进一步使 磨损显著降低至 56\%。团队还使用具有销盘旋转滑 动配置的高温摩擦测量仪(型号为 HT-1000)作为测 试仪器, 评估了铅-铋共晶液态金属的润滑性能, 这 种液态金属在核反应堆中作为主要冷却剂, 为模拟工 作环境, 试验是在氩气环境中来模拟真空环境的, 结果表明其有具有优异的润滑性, 试验过程中测得其 
工作的摩擦因数最低可达 0.1 。

考虑到滑动速度对润滑剂的减摩性能也有着直 接关系, 国内西安交通大学董光能教授团队 ${ }^{[21]}$ 使用 UMT-2 球盘式摩擦磨损试验机, 对于同种摩擦副材 料在不同频率下进行试验发现, 较高的滑动速度更 有利于液态金属发挥其减摩能力。并且团队将液态 金属与表面织构化相联系, 通过试验发现液态金属 可以明显降低纹理凹坑表面的摩擦因数和磨损率, 并且说明了这是因为纹理凹坑的存在有利于液态金 属储存和摩擦膜的沉积。

通过试验我们可以很容易地发现, 液态金属作 为润滑剂能够体现出一定的良好减摩性能, 但是这 些实验设置得相对简单, 当液态金属处在环境复杂 多变、时间较长的工作条件中时, 其减摩性能是否 会受影响尚不明确, 并且没有与其他传统润滑剂作 比较实验, 目前只是停留在发现其能够减小摩擦磨 损的阶段。

\section{2 液态金属的高温性能}

在实际日常生活中, 有相当一部分的机械传动 故障是由于温度过高, 导致发生了不可承受的摩擦 和磨损。并且黏度作为流体润滑剂的一个重要性能 参数, 与温度有着密不可分的关系, 这就要求在高 温条件下使用的润滑剂要有一定良好的高温性能和 导热性。

澳大利亚阿德莱德大学的 SARAFRAZ 和 ARJOMANDI ${ }^{[22]}$ 通过设计试验发现, 随着接触表面 倾斜角度的增加, 液态金属镓的热导率也会增加, 这是因为较高的倾斜角度会使镓在接触表面的流动 阻力减小, 从而流动性会加强, 液态金属的接触角 被确定为可能有效的传热特性参数。这对以后评价 液态金属的传热性能提供了方法与依据。

液态金属具有导电性, 所以当液态金属处于磁 场环境中时会产生感应电流, 感应电流会与磁场相 互作用产生洛伦兹力, 进而影响液态金属流动。考 虑到这种情况, 北京航天长征飞行器研究所的景昭 等 ${ }^{[23]}$ 研究了磁流体动力学效应下 $U$ 形管道中液态金 属的流动, 通过数值模拟结果发现流量进入 $\mathrm{U}$ 形流 道中心区域时形成螺旋状流动, 产生的摚拌作用可 能对管道内热交换有利。这也启发了一条新的思路, 即通过控制磁场、电场来控制液态金属的流动, 进 而促进热交换, 以适应高温工作环境。

中国科学院兰州化学物理研究所固体润滑国家 重点实验室的 LI 等 ${ }^{[24]}$ 同样适用 SRV-IV 型摩擦磨损 试验机进行了从 $-10{ }^{\circ} \mathrm{C}$ 到 $800{ }^{\circ} \mathrm{C}$ 宽温度范围内液 态金属的润滑性能试验, 结果发现在 $-10{ }^{\circ} \mathrm{C}$ 和 $20{ }^{\circ} \mathrm{C}$ 下, 液态金属表现出优异的摩擦学性能, 在
$800{ }^{\circ} \mathrm{C}$ 的高温下, 液态金属表现出有效的润滑性能。 此研究证明了液态金属能在较宽温度的范围内用作 润滑剂, 这对接下来提高机械运动部件寿命和其工 作性能具有重要意义。

常见液态金属的热导率一般为 $10 \sim 40 \mathrm{~W} /(\mathrm{m} \cdot \mathrm{K})$, 比传统的冷却工质水高出 2 个数量级, 并且液态金 属的黏度跟水在同一个量级, 因此也具有很好的流 动性 ${ }^{[25]}$, 再加上液态金属本身具有的优异的热物理 性能, 如高导热性、不易燃性、无毒性和低熔点, 这些特性都使得其在散热领域具有一系列新颖的应

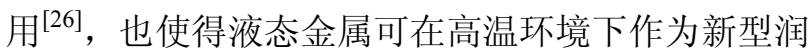
滑剂来使用。

\section{3 液态金属的承载性能}

随着载荷的增加, 两表面之间的油膜厚度会逐 渐减小, 当载荷增加到一定程度时, 连续的油膜会 被表面的峰顶破坏, 局部产生表面之间的直接接触, 润滑状态从液体润滑逐渐变为边界润滑。当载荷继 续不断增加时, 两表面会直接接触变为干摩擦, 产 生很大的磨损, 造成机械零部件的破坏。因此要求 润滑剂要有一定的承载性能。

在文献[18]中, 使用振荡摩擦试验机, 在 $1500 \mathrm{~N}$ 高负载和不同润滑剂的条件下, 研究了当使用陶瓷 球与钢盘作为摩擦对时液态金属的润滑性能。试验 表明，镓基液态金属不但在高负载下能够成功润滑 钢-瓷摩擦对, 甚至发现其润滑性能明显优于油和离 子液体。此试验证明了液态金属可在高载荷工作条 件下用作润滑剂的可能性。其实早在 1963 年就发 现对于使用液态金属润滑的轴颈轴承、推力轴承和 滑块轴承, 通过施加外部磁场和外部电流, 可以在 流体动力轴承上实现润滑膜承载能力的显著增 加 ${ }^{[27]}$, 但是如果只是通过单独施加磁场, 除了极 大的磁场外，不能实现明显的加压。由于当时科技 水平的限制, 只是提出了这种提高承载能力方法的 可行性。

润滑剂的承载能力与其黏度有很大的关系, 通 过改变润滑剂的导热性和热膨胀性可以改善润滑剂 的极压润滑性能。考虑到传统有机润滑剂的散热能 力有限, 而镓基液态金属有很高的热膨胀性, 清华 大学机械系摩擦学国家重点实验室的田显团队 [28] 使用标准的四球测试，对商用齿轮油、未配制的基 础油和镓基液态金属进行了比较试验, 通过标准四 球测试得知镓基液态金属可用于极压润滑, 并且利 用液态金属的高热效率实现了极压润滑的最新技 术，比一般有机润滑剂高 2 个数量级。

在国外有关 CT 球管的研究中还发现, 液态金 属的负载能力与流体速度有很大关系 ${ }^{[29-30]}$, 所以可 
以深入研究两者之间的关系, 从而通过控制工作转 速来控制流体速度, 进而优化液态金属的负载性能。

\section{4 液态金属的润湿性能}

润滑剂要充分发挥其润滑作用的前提就是润滑 剂能迅速而又牢固地润湿摩擦表面 ${ }^{[31]}$, 固液润湿性 强的界面油膜厚度较高, 而当固液界面润湿性较差 时,润滑膜厚度明显降低并且界面部分滑移导致润 滑膜厚度降低 ${ }^{[32]}$ 。所以在实际工作环境中, 润滑剂 的润湿性能要作为一个考量标准。

在文献[33]中, 通过使用 FLUENT 软件, 设置 液态金属与倾斜固壁面的接触角来模拟不同的润湿 情况, 以观察膜的流动情况。结果发现润湿性较差 时, 膜不易完全覆盖倾斜壁面底部, 易发展为溪状 流, 所以液态金属的润湿性能与润滑剂的表面张力 与接触角有很大关系。

液态金属具有较高的表面张力 ${ }^{[34], ~}$ 因此其润湿 性能有一定局限性, 但是美国北卡罗来纳州立大学 的 KHAN 等 ${ }^{[35]}$ 在镓液态金属的表面上通过使用电 化学沉积(或去除)氧化物时, 发现这种方法可以用 来控制镓液态金属的表面张力, 此方法使用非常低的 电压就使镓液态金属的表面张力从大约 $500 \mathrm{~mJ} / \mathrm{m}^{2}$ 明 显降低至接近零, 并且这种变化是完全可逆的。 另外考虑到液态金属不同于一般润滑剂的特殊性 能, 外加磁场时产生洛伦兹力, 进而起到阻碍作 用减慢流动速度, 也会增加润滑膜的覆盖区域。 虽然这种外加磁场、电场的方法只适用于特殊润 滑工作场合, 但也展现了液态金属作为特殊新型 润滑剂的可能。

另外在文献[22]中发现, 表面粗䊁度是控制镓 和镓-铋液态金属在表面上接触角的关键参数, 随着 表面粗粘度的增加, 镓和镓-铋液态金属的接触角增 大, 并且随着时间的推移, 镓的接触角减小并达到 恒定值, 这对液态金属的润湿性能研究提供了参考。 也可以在液态金属中添加其他成分, 以获得更好的 性能, 比如在镓液态金属中增加铋纳米颗粒, 会降 低分子间作用力，使得液态金属的润湿性增加。

\section{3 液态金属润滑机理的研究现状}

\section{1 液态金属的自身性能}

液态金属作为一种金属, 其导热性是更加优于 其他润滑剂的, 超散热性使得液态金属具有很好的 承载能力 ${ }^{[28]}$, 同时相较于固体润滑剂, 液态金属又 具有很好的流动性。这些固有物理化学属性都使得液 态金属可以在一些特定工作场合作为润滑剂来使用。
液态金属不同于其他一般润滑剂主要因为其本 质是一种金属, 具有导电性。随着 20 世纪 60 年代 核动力设备的发展, 出现了一种新型轴承, 即磁流 体轴承, 这种轴承在外加磁场的工作环境下, 以导 电流体作为润滑剂, 利用电磁感应力对流体运动的 阻碍作用, 来成倍增加流体的等效黏度, 从而优化 其承载性能, 目前多用于航空航天、卫星和潜艇制 导等尖端科学领域内。在常温下, 磁流体轴承多用 永做导电流体, 但是在 2017 年, 世界卫生组织国际 癌症研究机构公布了致癌物清单初步整理参考, 寽 和无机录化合物在 3 类致癌物清单中。而考虑到 $\mathrm{Ga}$ 在室温下为液体, 无毒环保不易燃, 并具有高导电 性, 所以 $\mathrm{Ga}$ 基液态金属可用于高温和核应用的冷 却系统中，同时 Ga-In-Sn 液态金属组分的低反应性 和无毒性等特质, 使其经常被用作或被认为是永的 潜在替代品 ${ }^{[36]}$, 在接下来的发展中镓基液态金属可 能会代替录广泛应于此领域。

早在 20 世纪 70 年代, 使用液态金属来冷却高 速增殖反应堆的项目已列入日本国家计划中, 设计 中的高速增殖反应堆使用液态钠作为冷却剂。同时 快中子反应堆是世界上第四代先进核能系统的首选 堆型, 代表了第四代核能系统的发展方向, 而液态 钠具有良好的物理化学性质: 钠原子很大, 中子碰 撞后质量不损失; 钠吸收中子不多, 不损耗能量; 导热性好, 解决反应堆过热问题; 钠的熔点低, 但 是沸点高达 $890{ }^{\circ} \mathrm{C}$, 在通常 $500 \sim 600{ }^{\circ} \mathrm{C}$ 的工作条件 下安全性高。所以液态钠是反应堆常用冷却剂。而在 此系统中如果用油作为与钠接触的轴承的润滑剂, 则 在高温中就会发生反应进而造成液态钠的污染, 并且 反应生成物有可能堵塞液态钠的流道, 所以, 在此系 统中, 轴承的润滑就不得不依靠钠液本身 ${ }^{[37]}$ 。

我们可以看出, 由于液态金属具有很多不同于 普通润滑剂的自身固有性能, 因此在一些特殊工作 场合可以作为润滑剂来使用。

\section{2 液态金属反应膜}

目前分析液态金属的润滑反应膜主要使用的方 法为: 通过使用显微仪器和能量色散谱来分析磨痕 的表面形貌和成分。尽管在电子显微镜下能够发现 材料表面有一层摩擦层的覆盖, 并且通过能量色散 谱可以得到摩擦层的构成元素, 但是对于这种摩擦 层的组成、结构和形成机理尚不清楚 ${ }^{[18]}$ 。

当 $\mathrm{Ga}_{65} \mathrm{In}_{24} \mathrm{Sn}_{12}$ 用于润滑 AISI 52100 轴承钢时, 能够分析出材料表面有一层摩擦膜一 $-\mathrm{FeGa}_{3}$, 这 种摩擦膜的熔点 $\left(824 \sim 860{ }^{\circ} \mathrm{C}\right.$ ) 明显低于钢 (大约 $\left.1300{ }^{\circ} \mathrm{C}\right)$, 并且纳米压痕表明这种摩擦膜在室温和 
高温下都比钢更柔顺, 猜测这种摩擦膜的低熔点和 相对较软的性质有助于它作为易剪切界面层, 并导 致低摩擦因数 ${ }^{[28]}$ 。但是在这个试验中没有得到在润 滑过程中形成的这种摩擦膜与减摩的直接关系, 为 探究这种直接关系, 刘维民院士团队 ${ }^{[38]}$ 将 T91 钢盘 浸入陶瓷坩埚的液态金属 $\mathrm{Ga}_{65} \mathrm{In}_{22} \mathrm{Sn}_{13}$ 中, 在高纯度 氩气条件下加热到 $600{ }^{\circ} \mathrm{C}$ 保持 $1 \mathrm{~h}$, 用这种方法形 成了 $\mathrm{FeGa}_{3}$ 膜, 又在 HT-1000 圆盘式摩擦磨损仪上 与 $\mathrm{Al}_{2} \mathrm{O}_{3}$ 球配对进行试验, 结果表明 $\mathrm{FeGa}_{3}$ 膜与钢 表面强烈结合, 在室温下有良好的机械和摩擦学性 能, 但在 $600{ }^{\circ} \mathrm{C}$ 时会增加磨损。这种简单的薄膜制 备方法可以保护应用于许多工业领域的铁基材料, 并且通过试验证明了 $\mathrm{FeGa}_{3}$ 膜的形成是液态金属体 现良好润滑性能的原因。

通过上述研究结果, 我们可以看出液态金属是 一种极易氧化的材料, 在两个摩擦表面间容易发生 反应形成摩擦膜, 而这种反应膜能够起到一定的减 摩作用，使得液态金属可以作为润滑剂使用。

\section{4 液态金属轴承的工作性能分析}

在机械传动系统中, 转动的轴系部件是必不可 少的部分, 但是由于存在质量不平衡问题, 在工作 过程中会产生振动, 振幅过大会导致轴承和转子的 结构损伤, 甚至影响机械结构的正常运转 ${ }^{[39]}$, 同时 对于机械设计而言, 良好静态特性是设计的基础, 而良好的动态性能是成功的关键 ${ }^{[40]}$ 。所以要考虑液 态金属轴承工作性能的分析。

目前对于液态金属轴承工作性能的研究较为成 熟的是快堆中所使用的钠洜。快堆是快中子增殖堆 的简称, 中国试验快堆是我国第一座快堆, 是我国 快中子增殖反应堆(快堆)发展的第一步, 其核热功 率 65 兆瓦, 试验发电功率 20 兆瓦, 是目前世界上为 数不多的具备发电功能的试验快堆。在 20 世纪 60 、 70 年代, 美国和俄罗斯的许多大型、高温旋转机械 中用到了液态金属钠, 文献[41]研究了 1969 年 11 月 1 日到 1970 年 4 月 30 日期间这些机械所用的液 态金属轴承, 考虑流体的惯性设计了计算程序, 整 理出了一份完整的报告和设计手册, 并设计试验篎 选合适的轴承表面材料, 此报告详细地介绍了液态 金属轴承的相关技术。中国试验快堆中使用的冷却 剂是高温液态金属钠, 其中有一关键设备, 即一回 路钠循环百, 在文献[12]中利用转子轴承系统动力 学建立了主轴的数学模型, 用以描述洜轴系的弯曲 振动, 并对泵机组进行了较系统的动力分析, 使用 影响系数法确定了钠原的临界转速, 并设计试验进
行了振动测试, 试验表明两台一回路钠循环泵运转 正常, 此工作为接下来对钠葲动态特性的研究奠定 了基础。另外在一般核电站的二回路主循环系统中, 钠泵流体动静压混合润滑轴承(简称钠泵轴承)是很 重要的组成部分, 国内上海交通大学陈汝刚等 ${ }^{[42]}$ 就钠 洜轴承的润滑机制和动力学特性进行了研究, 结果发 现: 随着载荷的增大, 钠洜轴承主要是静压效应起主 导作用, 动压效应较小, 几乎可以忽略; 但是当继续 增大外载荷时, 钠百轴承液膜的动压效应逐渐增强。 目前这种洜都采用静力式钠润滑轴承, 使用静力式轴 承主要是为了形成液态钠的润滑膜, 并且必须选择好 轴和轴承的形状及其间隙大小, 也要选择好不至发生 磨损和咬卡现象的轴和轴承零件的配合 ${ }^{[42]}$ 。

医疗 X 射线的产生主要关乎两个部件，即发射 电子束的阴极部件与接收电子束的阳极部件, 电子 束打在阳极上只有 $1 \%$ 的能量转化为 $\mathrm{X}$ 射线, 其余 绝大部分转化成热量释放, 当阳极为固定时, 热量 集中在一个点会造成材料的局部损伤, 同时随着医 学影像技术的发展和 $\mathrm{CT}$ 检查的普及, $\mathrm{CT}$ 球管作为 $\mathrm{CT}$ 机的一个昂贵的消耗部件, 市场需求快速增长, 目前如何提高其工作性能问题引起广泛关注与研究。 目前发展成熟的一个技术即用旋转阳极代替固定阳 极, 将热量集中于一点变为热量分散成一个弧线区 域, 避免材料的局部损伤。考虑到工作条件, 要求旋 转阳极所使用的润滑剂不可污染真空管、要承受高 温、能够导电, 最好能够导热, 因此镓基液态金属成 了最好的选择。欧美国家对这种液态金属已进行了详 细研究并发表了多个专利 ${ }^{[43-46]}$ 。如今国内西安交通大 学陈渭教授的团队 ${ }^{[47]}$ 就旋转阳极所用的液态金属润 滑螺旋槽轴承做了详细的动力学分析(图 1): 螺旋槽轴 承沟槽的倾斜结构, 在直角坐标系下计算可能会导致 很大的误差, 所以将直角坐标转化成斜坐标重新推导 雷诺方程; 考虑到实际工作为高速流动状态, 镓基液 态金属的黏度在室温时约为水黏度的两倍 ${ }^{[48]}$, 所以工 作时的流动形式为紊流; 镓基液态金属在轴承材料上 的接触角大、润湿性不佳, 所以文献中又考虑了边界

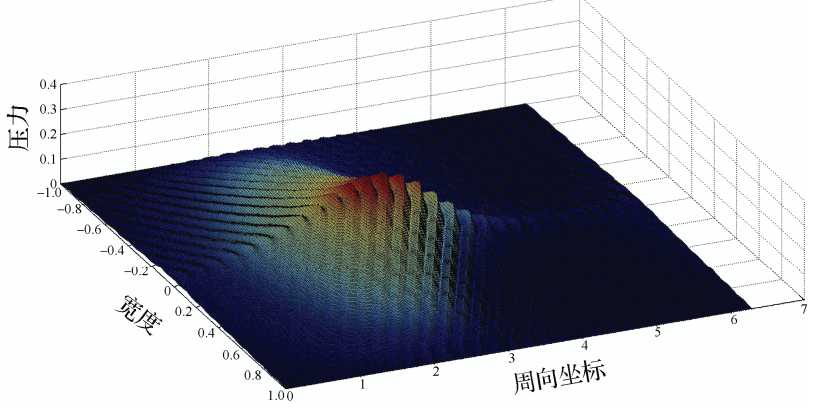

图 1 人字槽径向轴承压力分布(量纲一) 
滑移问题; 综合以上实际考虑进行了计算, 通过软件 仿真得出相应工作条件下螺旋槽轴承的最佳结构, 以获得比较良好的静动态性能。此研究是目前国内 最早研究液态金属润滑螺旋槽轴承的项目, 对接下 来 $\mathrm{CT}$ 球管的工作性能优化有很重要的意义。

\section{5 液态金属用作润滑剂的发展展望}

目前液态金属作为润滑剂应用于实践的场合还 很少, 但是这些应用都是在真空条件下进行, 比如 旋转阳极的 CT 球管用液态金属作为润滑剂的工作 条件为真空 ${ }^{[49]}$, 并且铅-铋共晶液态金属用于先进液 态金属核反应堆中的工作条件也为真空 ${ }^{[19]}$ 。在许多 研究试验中也是模拟真空环境或者在真空环境下进 行的, 比如在氩气和高真空环境下进行试验发现镓 基液态金属在非氧化条件下可以有效地作为螺旋槽 轴承的可靠润滑剂 ${ }^{[00]}$, 并且将真空条件与非真空条 件做对比试验发现由镓扩散膜获得的摩擦和磨损在 真空中比在空气中低 ${ }^{[8]}$, 美国爱荷华州立大学的 CUTINHU 等 ${ }^{[51]}$ 研究发现表面氧化会改变液态金属 颗粒的表面纹理进而改变表面粗楉度。目前很多试 验已经表明液态金属的氧化性是不容忽视的, 这可 能会对液态金属的润滑性能产生很大的影响, 所以 如何控制液态金属的这种氧化性以获得最佳润滑性 能, 是接下来要重点研究的内容。

考虑到研究液态金属中的空蚀对于工业机械的 安全性非常重要, 福井大学的 HARRORI 等 ${ }^{[52-53]}$ 开 发了一种新的测试装置, 可以在 $50 \sim 400{ }^{\circ} \mathrm{C}$ 的温度 下进行空化腐蚀测试, 并试验发现铅铋合金液态金 属的空化腐蚀速率随着温度的升高而增大, 并计算 出, 在 $10 \sim 40{ }^{\circ} \mathrm{C}$ 下每增加 $1{ }^{\circ} \mathrm{C}$ 腐蚀率增加 $3 \%$ $4 \%$, 在 $40 \sim 50{ }^{\circ} \mathrm{C}$ 下每增加 $1{ }^{\circ} \mathrm{C}$ 腐蚀率增加 6\% $7 \%$ 。此研究中开发的新的测试装置可以用来测量其 他液态金属的空化腐蚀性, 并且可以根据试验结果 来控制工作温度, 以发挥液态金属的最佳性能。中 国科学院理化技术研究所低温重点试验室的崔云涛 等 ${ }^{[54]}$ 研究了液态金属腐蚀对三种常规金属合金(包 括 T2 铜, 304 不锈钢和阳极氧化 6061 铝合金)在 100 400 ${ }^{\circ} \mathrm{C}$ 范围内热物理性能的影响, 结果发现 T2 铜和 304 不锈钢在液态共晶镓铟中都会出现明显 的导热率降低, 阳极氧化 6061 铝合金具有更好的耐 腐蚀性, 但是只有经过一定的良好表面处理后, 阳 极氧化 6061 铝合金才能用作液态金属系统的结构 材料。所以当使用液态金属作为润滑剂时, 必须考 虑液态金属的腐蚀性, 选择合适的材料制造零部件, 使其与液态金属配合使用, 发挥最大效果。但在实
际工作中，液态金属的腐蚀过程是很复杂多变的， 仍然存在很多问题有待于研究,在文献[55]中就提 到, 关于合金化元素对固态金属在液态金属中的溶 解度及两者之间化学相容性的影响机制、合金元素 在液态金属中的扩散行为对腐蚀行为的影响规律、 当工作过程中发生磨损等机械损伤时的腐蚀过程及 机制等有关液态金属的腐蚀性的研究还有大量的工 作要做。

清华大学的田显团队 ${ }^{[56]}$ 试着将镓基液态金属 加入润滑脂中，结果发现加入液态金属的润滑脂的 极压润滑性能明显提高。此项研究成果这也为我们 提供了一个新的思路, 即将液态金属与其他润滑剂 进行混合使用, 以得到两者综合的润滑性能。目前 有很多关于向液态金属中添加其他元素的试验研 究, 并发现在添加了其他元素后, 原液态金属的物 理化学性质发生了改变: 当向 Ga-Sn-Zn 液态合金中 添加 $\mathrm{Pb}$ 时, 会使得液态金属的密度、黏度和热电功 率增加, 同时也会导致液态金属的表面张力、电导 率和导热率的降低 ${ }^{[57]}$; 向共晶 Ga-Sn-Zn 液态合金 中添加 $\mathrm{Bi}$ 会导致液态金属的黏度, 密度和热电功率 增加, 同时降低液态金属的导电率, 热导率和表面 张力 ${ }^{[58]}$; 添加 $\mathrm{Sn}$ 可能会提高液态 $\mathrm{Ga}-\mathrm{Pb}$ 合金的润 湿温度, 且 $\mathrm{Ga}-\mathrm{Pb}$ 二元液态合金的表面能可以通过 添加表面活性第三元素溶质来改变 ${ }^{[59]}$ 。所以我们可 以根据不同的要求, 向液态金属中添加其他元素, 使其更加适应所处的工作环境。

在进行液态金属用作润滑剂的理论与仿真分析 工作时, 要格外注意液态金属与传统润滑剂不同的 独特的物理性质，镓基液态金属的黏度非常小，所 以在研究其在高速运动情况下的润滑性能时, 选取 的流体动力润滑模型应为紊流的形式, 并且由于其 在常用轴承材料上的接触角比较大, 体现出的润湿 性有一定的局限, 所以在实际应用分析中还要考虑 边界滑移的问题。这些与传统润滑剂不同的物理性 质就要求研究工作者在进行理论与仿真分析工作 时, 必须认真仔细地处理分析工作中所用模型的建 立与选取。

液态金属表现出良好润滑性能的同时，本身还 具有非常好的冷却性能和导电性能 ${ }^{[26]}$, 这就使得液 态金属可以适应其他多变的复杂环境。但是目前对 于液态金属黏滞性的研究偏重于理论研究和宏观研 究 ${ }^{[60]}$, 若能对其微观进行深入研究, 对进一步挖掘 液态金属的润滑性能具有重要的技术指导意义。并且 我们已经知道润滑介质在材料上的润湿性与润滑膜 的形成有着直接关系, 但是目前对液态金属反应性润 


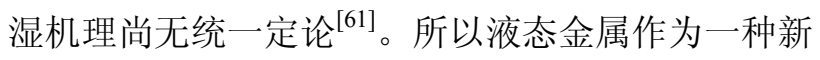
型润滑剂, 还有很多需要我们去深入研究的地方。

\section{6 结论}

通过以上综述我们可以看出, 随着社会的不断 进步和科技的不断提高, 发展出了各种各样的新行业 和新技术, 对于机械系统中润滑剂的性能也有了更高 的新的要求, 而各界对于液态金属用作润滑剂的研究 是不断深入和细致的, 目前的试验与研究已经证明了 液态金属的出现为满足这些要求提供了可能。

（1）液态金属可以有效降低摩擦磨损，当镓基 液态金属作为载流润滑剂时, 与无电流对比可以进 一步使磨损显著降低至 $56 \%$; 铅-铋共晶液态金属 在氩气环境中的摩擦因数大约为 0.1 。

（2）液态金属可用于很宽的温度范围, 在 $-10{ }^{\circ} \mathrm{C}$ 下仍能表现出优异的摩擦学性能, 并且在 $800{ }^{\circ} \mathrm{C}$ 的高温下能够表现出有效的润滑性能。

（3）液态金属具有很好的极压性能, 镓基液态 金属在 $1500 \mathrm{~N}$ 高负载条件下能够成功润滑钢-瓷摩 擦对, 并且通过标准四球测试可知镓基液态金属可 用于极压润滑。

(4) 液态金属可以作为一种添加剂与其他润滑 剂混合, 也可以向液态金属中添加其他元素, 使其 性能更满足工作要求。

（5）液态金属由于其独特的物理化学性质可以 用于电、磁和热等场合。

我国进行液态金属作为润滑剂的研究起步较 晚, 国外一些液态金属轴承技术已发展很成熟, 将 来我国对于液态金属用作润滑剂的研究应着重从如 下几方面努力发展。

（1）液态金属具有氧化性, 而与摩擦材料发生 反应产生的摩擦膜有一定润滑作用, 接下来可以尝 试设计液态金属的氧化反应, 极大提高其润滑性能。

（2）液态金属具有腐蚀性，目前有关液态金属 腐蚀机理的研究还存在很多亟需解决的问题, 所以 如何控制液态金属的腐蚀作用是接下来要进一步研 究的内容。

(3) 液态金属会与其他材料发生反应, 这种反 应造成的润滑机理目前尚无统一定论, 仍需要深入 调查研究。

(4) 润滑剂的剩余使用寿命与其工作性能密切 相关, 但是目前关于液态金属剩余使用寿命的研究 仍是空白, 如果要使液态金属作为润滑剂广泛应用 于实际中, 此研究必不可少。

（5）液态金属用作润滑剂的理论与仿真分析较
少, 将来可以加深对此方面的研究, 建立起一套对 液态金属普遍使用的理论与仿真体系。

\section{参 考 文 献}

[1] 刘志远, 刘静. 使中国液态金属研究走在世界前列 [J]. 科技导报，2016，34(4): 96-100.

LIU Zhiyuan. LIU Jing: Making China's liquid metals research in the forefront of the world[J]. Science and Technology Review，2016，34(4): 96-100.

[2] 于永泽, 刘静. 液态金属 3D 打印技术进展及产业化前 景分析 [J]. 工程研究-跨学科视野中的工程, 2017, 39(6): 577-585.

YU Yongze, LIU Jing. Progress in liquid metal 3D printing technology and analysis of industrialization prospects[J]. Journal of Engineering Studies, 2017, 39(6): 577-585.

[3] 姚飞. 液态金属的应用 [J]. 科技视界, 2019(5)： 129-131.

YAO Fei. The application of liquid metal[J]. Science \& Technology Visin, 2019 (5): 129-131.

[4] ZHANG Jie, YAO Youyou, SHENG Lei, et al. Selffueled biomimetic liquid metal mollusk[J]. Advanced materials (Deerfield Beach, Fla.), 2015, 27(16): 2648-2655.

[5] WANG Hongzhang, YAO Youyou, WANG Xiangjiang, et al. Large-magnitude transformable liquid-metal composites[J]. ACS Omega, 2019, 4(1): 2311-2319.

[6] 刘维民, 许俊, 冯大鹏, 等. 合成润滑油的研究现状及 发展趋势[J]. 摩擦学学报, 2013，33(1): 91-104.

LIU Weimin, XU Jun, FENG Dapeng, et al. The research status and prospect of synthetic lubricating oils[J]. Tribology, 2013, 33(1): 91-104.

[7] 石灵, 张富治, 王瑞海, 等. 医用 CT 球管国内外现状 及发展趋势[J]. 真空电子技术, 2018(2): 61-68.

SHI Ling, ZHANG Fuzhi, WANG Ruihai, et al. Domestic and foreign situation and development trend of medical CT X-Ray tubes[J]. Vacuum Electronics, 2018(2): 61-68.

[8] BUCKLEY D H, JOHNSON R L. Gallium-rich films as boundary lubricants in air and in vacuum to $10-9 \mathrm{~mm}$ Hg[J]. ASLE Transactions, 1963, 6(1): 1-11.

[9] KOStOMOV A G, ZOZUlYA V D, SIN' KOV V A, et al. Bearing materials based on fibers and liquid-metal lubricants $[\mathrm{J}]$, Soviet Powder Metallurgy \& Metal Ceramics, 1973, 12(7): 534-537.

[10] BURTON R G, BURTON R A. Gallium alloy as lubricant 
for high current density brushes[J]. IEEE Transactions on Components, Hybrids, and Manufacturing, Technology, 1988, 11(1): 110-115.

[11] PERSAD C, YEOH A, PRABHU G, et al. On the nature of the armature-rail interface: Liquid metal effects[J]. IEEE Transactions on Magnetics, 1997, 33(1): 140-145.

[12] 董碧波. 中国试验快堆一回路钠循环泵振动特性分析 [J]. 核科学与工程, 2013, 33(3): 231-237.

DONG Bibo. Analysis on vibration characteristics of the primary sodium pump of china experimental fast reactor[J]. Nuclear Scence and Engineering, 2013，33(3): 231-237.

[13] 秦琴, 刘宜伟, 王永刚, 等. 基于液态金属的柔性导线 的制备方法研究进展 [J]. 电子元件与材料, 2017 , 36(04): 1-8.

QIN Qin, LIU Yiwei, WANG Yonggang, et al. Recent progress of methods for fabricating flexible conductive wires based on liquid metals[J]. Electronic Components and Materials, 2017, 36(04): 1-8.

[14] REGAN M J, TOSTMANN H, PERSHAN P S, et al. X-ray study of the oxidation of liquid-gallium surfaces[J]. Physical Review B, 1997, 55(16): 10786-10790.

[15] ZHENG Yi, HE Zhizhu, GAO Yunxia, et al. Direct desktop printed-circuits-on-paper flexible electronics[J]. Scientific Reports， 2013， 3: 1786.

[16] DOBOSZ A, PLEVACHUK Y, SKLYARCHUK V, et al. Thermophysical properties of the liquid $\mathrm{Ga}-\mathrm{Sn}-\mathrm{Zn}$ eutectic alloy[J]. Fluid Phase Equilibria, 2018, 465: 1-9.

[17] GUO Jie, CHENG Jun, TAN Hui, et al. Ga-based liquid metal: A novel current-carrying lubricant[J]. Tribology International, 2019, 135: 457-462.

[18] CHENG Jun, YU Yuan, GUO Jie, et al. Ga-based liquid metal with good self-lubricity and high load-carrying capacity[J]. Tribology International, 2019, 129: 1-4.

[19] CHENG Jun, ZHU Shengyu, TAN Hui, et al. Lead-bismuth liquid metal: Lubrication behaviors[J]. Wear, 2019, 430-431: 94-99.

[20] 程军, 于源, 朱圣宇, 等. 多功能室温液态金属在不同 摩擦副条件下的润滑性能研究[J]. 摩擦学学报, 2017, 37(4): 435-441.

CHENG Jun, YU Yuan, ZHU Shengyu, et al. Lubrication characteristics of multifunctional lliquid-state metal materials under different sliding-pairs[J]. Tribology, 2017, 37(4): 435-441.

[21] LI Xing, LI Yihong, TONG Zhe, et al. Enhanced lubrication effect of gallium-based liquid metal with laser textured surface[J].. Tribology International, 2019, 129: 407-415.

[22] SARAFRAZ M M, ARJOMANDI M. Contact angle and heat transfer characteristics of a gravity-driven film flow of a particulate liquid metal on smooth and rough surfaces[J]. Applied Thermal Engineering, 2019, 149: $602-612$

[23] 景昭, 杨驰, 曹宇清, 等. 磁场作用下 $U$ 形流道中液 态金属流动的数值模拟 [J]. 山东化工，2017，46(13): 155-157.

JING Zhao, YANG Chi, CAO Yuqing, et al. Numerical simulation for liquid metal flow in a U-bend under a magnetic field[J]. Shandong Chemical Industry, 2017, 46(13): 155-157.

[24] LI Yi, ZHANG Songwei, DING Qi, et al. Liquid metal as novel lubricant in a wide temperature range from -10 to $800{ }^{\circ} \mathrm{C}[\mathrm{J}]$. Materials Letters, 2018, 215.

[25] DENG Yueguang, LIU Jing. An experimental investigation of liquid metal thermal joint[J]. Energy Conversion and Management, 2012, 56: 152-156.

[26] 杨小虎, 刘静. 液态金属高性能冷却技术: 发展历程与 研究前沿[J]. 科技导报，2018，36(15): 54-66.

YANG Xiaohu, LIU Jing. Liquid metal high-performance cooling technology: Development history and research frontier[J]. Science and Technology Guide, 2018, 36(15): 54-66.

[27] HUGHES W F. Magnetohydrodynamic lubrucation and application to liquid metals[J]. Industrial Lubrication and Tribology, 1963, 15(3): 125-133.

[28] LI Haijiang, TIAN Pengyi, LU Hongyu, et al. State-of-the-art of extreme pressure lubrication realized with the high thermal diffusivity of liquid metal[J]. ACS Applied Materials \& Interfaces, 2017, 9(6): 5638-5644.

[29] HARDING G, THRAN A, DAVID B. Liquid metal anode $\mathrm{X}$-ray tubes and their potential for high continuous power operation[J]. Radiation Physics and Chemistry, 2003, 67(1): 7-14

[30] DAVID B R, BARSCHDORF H, DMMRMANN V, et al. Liquid-metal anode X-ray tube[J]. Proceedings of SPIE- The International Society for Optical Engineering, 2004, 5196: 432-443.

[31] 刘芳, 李杰, 时凯. 润滑剂与润湿现象 [J]. 聚氯乙烯, 2009, 37(6): 21-25, 28.

LIU Fang, LI Jie, SHI Kai. Lubricants and wetting phenomena[J]. Polyvinyl Chloride, 2009, 37(6): 
$21-25,28$.

[32] 杨淑燕, 郭峰, 马冲, 等. 固液润湿性对流体动压润滑 薄膜的影响 [J]. 摩擦学学报, 2010, 30(2): 203-208. YANG Shuyan, GUO Feng, MA Chong, et al. Influences of the liquid solid wettability on thin hydrodynamic lubrication films[J]. Tribology, 2010, 30(2): 203-208.

[33] 张秀杰, 潘传杰, 许增裕. 润湿性及磁场对液态金属自 由表面膜流流动状态的影响 $[\mathrm{J}]$. 核聚变与等离子体物 理, 2017, 37(1): 52-57.

ZHANG Xiujie, PAN Chuanjie, XU Zengyu. Effect of wettability and magnetic field on flow state of liquid metal free surface membrane[J]. Nuckear Fuson and Plasma Physics, 2017, 37(1): 52-57.

[34] YUAN Bin, HE Zhizhu, FANG Wenqiang, et al. Liquid metal spring: oscillating coalescence and ejection of contacting liquid metal droplets[J]. Science Bulletin, 2015, 60(6): 648-653.

[35] KHAN M R, EAKER C B, BOWDEN E F, et al. Giant and switchable surface activity of liquid metal via surface oxidation[J]. Proceedings of the National Academy of Sciences, 2014, 111(39): 14047-14051.

[36] PLEVACHUK Y, SKLYARCHUK V, ECKERT S, et al. Thermophysical properties of the liquid Ga-In-Sn eutectic alloy[J]. Journal of Chemical \& Engineering Data, 2014, 59(3): 757-763.

[37] 下屋敷重广, 伊藤喜夫, 王庆春. 液态金属冷却高速增 殖反应堆用机械式钠泵 $[J]$. 化工与通用机械, 1973(6): 49-54.

XIAWU Shizhongguang, YITENG Xifu, WANG Qingchun. Mechanical sodium pump for liquid metal colling high-Speed proliferation reactor[J]. Chemical and General Machinery, 1973(6): 49-54.

[38] GUO Jie, CHENG Jun, WANG Shuai, et al. A Protective $\mathrm{FeGa}_{3}$ film on the steel surface prepared by in-situ hot-reaction with liquid metal[J]. Materials Letters, 2018, 228: $17-20$.

[39] 李苗苗, 李卓, 马亮亮, 等. 轴承配置对转子轴系振动 特性的影响[J]. 航空动力学报, 2019, 34(6): 1209-1216. LI Miaomiao, LI Zhuo, MA Liangliang, et al. Influence of bearing arrangement on vibration characteristics of rotor shaft system[J]. Journal of Aerospace Power, 2019, 34(6): 1209-1216.

[40] 李蓓智, 吴喜如, 杨建国. 精密数控车床电主轴-轴承转子系统动态性能计算分析 $[\mathrm{J}]$. 机械设计与制造, 2010(3): 173-175.
LI Peizhi, WU Xiru, YANG Jianguo. Analysis and calculation on dynamic characteristics of precision $\mathrm{NC}$ lathe motorized spindle system[J]. Machinery Design\& Manufacture, 2010(3): 173-175.

[41] SMALLEY A J, GEREN B F, ARWAS E B. Liquid metal bearings technology for large, high-temperature sodium rotating machinery[R]. Progress Report, November 1, 1969-April 31, 1970. 1971.

[42] 陈汝刚, 解忠良, 饶柱石, 等. 二回路系统钠洜流体动 静压混合润滑轴承的润滑机制及动力学特性 [J]. 润滑 与密封, 2017, 42(8): 6-13.

CHEN Rugang, XIE Zhongliang, RAO Zhushi, et al. Lubrication regime and dynamic characteristics of hydro-hybrid bearing of two-circuit main loop Na liquid pump system[J]. Lubrication Engineering, 2017, 42(8): 6-13.

[43] GERKEMA, JAN, PELZER, JOZEF B. Metal-lubricated helical-groove bearing comprising an anti-wetting layer: Europe, EP84201596. 8[P]. 1985-05-15.

[44] BITTL H. X-ray tube with liquid-metal fluid bearing: America, US10828577[P]. 2004-04-21.

[45] PINERA A, PHILIP C, PELFREY. Hydrodynamic liquid metal bearing: America, US11799651[P]. 2007-05-02.

[46] TURAGA R, PARAMPIL G. Bearing mechanism and X-ray tube: America, US11481091[P]. 2006-07-05.

[47] 李亚倩, 杨攀, 陈渭. 考虑紊流和滑移影响的液态金属 润滑螺旋槽轴承设计研究 $[\mathrm{J}]$. 西安交通大学学报, 2019, 53(8): 15-23.

LI Yaqian, YANG Pan, CHEN Wei. A study of bearing design for liquid metal lubricated spiral grooves under influences of turbulence and $\operatorname{slip}[\mathrm{J}]$. Journal of $\mathrm{Xi}$ 'an Jiaotong University, 2019, 53(8): 15-23.

[48] PROKHORENKO V Y, ROSHCHUPKIN V V, POKRASIN M A, et al. Liquid gallium: Potential uses as a heat-transfer agent [J]. High Temperature, 2000, 38(6): 54-968.

[49] HATTORI H, FUKUSHIMA H, YOSHI Y, et al. Proposal of a high rigidity and high speed rotating mechanism using a new concept hydrodynamic bearing in $\mathrm{X}$-Ray tube for high speed computed tomography[J]. Joumal of Advanced Mechanical Design, Systems, and Manufacturing, 2009, 3(1): 105-114.

[50] GERKEMA J. Gallium-based liquid-metal full-film lubricated journal bearings[J]. ASLE Transactions, 1985, 28(1): 47-53. 
[51] CUTINHU J, CHANG B S, OYOLA R S, et al. Autonomous thermal-oxidative composition inversion and texture tuning of liquid metal surfaces[J]. ACS Nano, 2018, 12(5): 4744-4753.

[52] HARRORI S, NIMURA A, YADA H. Effect of temperature on cavitation erosion of $9 \mathrm{Cr}$ steel in liquid metal[J]. Wear, 2013, 303(1-2): 269-275.

[53] HARRORI S, YADA H, KURACHI H, et al. Effect of liquid metal composition and hydrodynamic parameters on cavitation erosion[J]. Wear, 2009, 267(11): 2033-2038.

[54] CUI Yuntao, DING Yujie, XU Shuo, et al. Liquid metal corrosion effects on conventional metallic alloys exposed to eutectic Gallium-Indium alloy under various temperature states[J]. International Journal of Thermophysics, 2018, 39(10): 113.

[55] 刘树勋, 李培杰, 曾大本. 液态金属腐蚀的研究进展 $[\mathrm{J}]$. 腐蚀科学与防护技术，2001，13(5)：275-278.

LIU Shuxun, LI Peijie, ZENG Daben. Research progress of liquid metal induced corrosion[J]. Corrosion Science and Protection Technology, 2001, 13(5): 275-278.

[56] BAI Pengpeng, LI Shaowei, TAO Dashuai, et al. Tribological properties of liquid-metal galinstan as novel additive in lithium grease[J]. Tribology International, 2018, 128: 140-143.

[57] DOBOSZ A, PLEVACHUK Y, SKLYARCHUK V, et al. Liquid metals in cooling systems: Experimental design of thermophysical properties of eutectic Ga-Sn-Zn alloy with $\mathrm{Pb}$ additions[J]. Journal of Molecular Liquids, 2019, 281: $542-548$

[58] DOBOSZ A, PLEVACHUK Y, SKLYARCHUK V, et al. Liquid metals in high-temperature cooling systems: The effect of $\mathrm{Bi}$ additions for the physicochemical properties of eutectic Ga-Sn-Zn[J]. Journal of Chemical \& Engineering Data, 2019, 64: 404-411.

[59] SERRE C, CHATAIN D, MURIS M, et al. Surface energy, adsorption, and wetting transitions in ternary liquid alloys $[\mathrm{J}]$. Metallurgical and Materials Transactions A (Physical Metallurgy and, Materials Science), 2001, 32(11): 2851-2858.

[60] 刘燕, 耿浩然, 孙民华，等. 液态金属黏滞性的研究现 状与展望[J]. 铸造, 2000，49(12)：875-878.

LIU Yan, GENG Haoran, SUN Minhua, et al. Present status and future development of the research on liquid metal viscosity[J]. Foundry, 2000，49(12): 875-878.

[61］魏芳. 液态金属在石墨表面润湿的研究现状 [J]. 甘肃 科技，2019，35(1): 32-35.

WEI Fang. Research status of wetting of liquid metal on graphite surface[J]. Gansu Science and Technology, 2019, 35(1): 32-35.

作者简介: 徐明宇, 女, 1996 年出生。主要研究方向为液态金属轴承。 E-mail: xumingyuwang@qq.com 陈渭(通信作者), 男, 1957 年出生, 博士, 教授, 博士研究生导师。主 要研究方向为轴承-转子动力学、流体润滑和多体系统动力学。

E-mail: chenw@mail.xjtu.edu.cn 